\title{
Palladium-Catalyzed, Carboxylic Acid-Assisted Allylic Substitution of Carbon Nucleophiles with Allyl Alcohols as Allylating Agents in Water
}

\author{
Kei Manabe and Shū Kobayashi \\ Graduate School of Pharmaceutical Sciences, The University of Tokyo \\ Hongo, Bunkyo-ku, Tokyo 113-0033, Japan
}

\section{Supporting Information}

\section{Experimental}

\section{General}

IR spectra were measured with JASCO FT/IR-610 spectrometers. NMR spectra were recorded on a JEOL JNM-LA300 or JNM-LA400 spectrometer. Tetramethylsilane $(\delta=0)$ was used as an internal standard for ${ }^{1} \mathrm{H}$ NMR and $\mathrm{CDCl}_{3}(\delta=77.0)$ for ${ }^{13} \mathrm{C}$ NMR. HRMS was performed by using a JEOL SX-102. Preparative thin-layer chromatography (TLC) was carried out using Wakogel B-5F. Structures of $\mathbf{3 a},{ }^{[1]} \mathbf{3 c},{ }^{[2]} \mathbf{3 g},{ }^{[3]}$ and $\mathbf{3} \mathbf{h}^{[4]}$ were confirmed by comparison with published spectral data.

\section{Typical Procedure of Allylic Substitution in Water (Table 1, entry 15)}

Cinnamyl alcohol (2a, $0.44 \mathrm{mmol})$ and dibenzoylmethane (1a, $0.29 \mathrm{mmol})$ were suspended in water $(1.8 \mathrm{~mL})$ at $\mathrm{rt}$ under argon, and then 1-adamantanecarboxylic acid $(0.029 \mathrm{mmol})$ and $\mathrm{Pd}\left(\mathrm{PPh}_{3}\right)_{4}(0.0059 \mathrm{mmol})$ were added. The whole was heated under reflux conditions in an oil bath (oil bath temperature: $115^{\circ} \mathrm{C}$ ) for $15 \mathrm{~min}$. After the mixture was cooled to rt, water $(5$ $\mathrm{mL})$ and brine $(5 \mathrm{~mL})$ were added. The organic materials were extracted with ethyl acetate, dried over sodium sulfate, passed through a short pad of silica gel, and concentrated under vacuum. To the resulting mixture, anthracene $(0.066 \mathrm{mmol})$ as an internal standard was added, and the NMR yield (98\%) was determined by ${ }^{1} \mathrm{H}$ NMR integration. The mixture was purified by silica gel preparative TLC (benzene) to give 3a in $87 \%$ isolated yield. 


\section{Ethyl 2-benzoyl-2-methyl-5-phenylpent-4-enoate (3b)}

Cinnamyl alcohol (2a, $0.36 \mathbf{m m o l})$ and ethyl 2-benzoylpropionate (1b, $0.24 \mathrm{mmol})$ were suspended in water $(1.4 \mathrm{~mL})$ at $\mathrm{rt}$ under argon, and then 1-adamantanecarboxylic acid (0.024 mmol $)$ and $\mathrm{Pd}\left(\mathrm{PPh}_{3}\right)_{4}(0.012 \mathrm{mmol})$ were added. The whole was heated under reflux conditions in an oil bath (oil bath temperature: $115^{\circ} \mathrm{C}$ ) for $5 \mathrm{~h}$. After the mixture was cooled to $\mathrm{rt}$, water $(5 \mathrm{~mL})$ and brine $(5 \mathrm{~mL})$ were added. The organic materials were extracted with ethyl acetate, dried over sodium sulfate, passed through a short pad of silica gel, and concentrated under vacuum. The mixture was purified by silica gel preparative TLC $($ AcOEt/hexane $=1 / 10)$ to give $\mathbf{3 b}$ in $90 \%$ isolated yield $(69 \mathrm{mg}) .{ }^{1} \mathrm{H}$ NMR $(400 \mathrm{MHz}$, $\left.\mathrm{CDCl}_{3}\right) \delta 1.05(3 \mathrm{H}, \mathrm{t}, J=7.1 \mathrm{~Hz}), 1.57(3 \mathrm{H}, \mathrm{s}), 2.87(1 \mathrm{H}, \mathrm{dd}, J=14.2,7.6 \mathrm{~Hz}), 2.95(1 \mathrm{H}, \mathrm{dd}$, $J=14.2,7.6 \mathrm{~Hz}), 4.09-4.15(2 \mathrm{H}, \mathrm{m}), 6.08(1 \mathrm{H}, \mathrm{dt}, J=15.8,7.6 \mathrm{~Hz}), 6.38(1 \mathrm{H}, \mathrm{d}, J=15.8$ $\mathrm{Hz}), 7.18-7.21(1 \mathrm{H}, \mathrm{m}), 7.25-7.31(4 \mathrm{H}, \mathrm{m}), 7.42(2 \mathrm{H}, \mathrm{t}, J=7.3 \mathrm{~Hz}), 7.53(1 \mathrm{H}, \mathrm{t}, J=6.8 \mathrm{~Hz})$, $7.85(2 \mathrm{H}, \mathrm{d}, J=7.3 \mathrm{~Hz}) ;{ }^{13} \mathrm{C} \mathrm{NMR}\left(100 \mathrm{MHz}, \mathrm{CDCl}_{3}\right) \delta 13.83,21.25,40.35,57.27,61.40$, $124.21,126.20,127.30,128.44,128.48,128.56,132.69,133.95,135.60,137.12,173.73$, 197.28; IR (neat) 2981, 1733, 1683, $1240 \mathrm{~cm}^{-1}$; HRMS (EI), calcd. for $\mathrm{C}_{21} \mathrm{H}_{22} \mathrm{O}_{3}: 322.1569$, found: 322.1581 .

\section{Ethyl 2-oxo-1-(3-phenyl-2-propen-1-yl)-1-cyclopentanecarboxylate (3c)}

Cinnamyl alcohol (2a, $0.34 \mathrm{mmol})$ and ethyl 2-oxocyclopentane-1-carboxylate (1c, 0.22 mmol) were suspended in water $(1.3 \mathrm{~mL})$ at $\mathrm{rt}$ under argon, and then 1-adamantanecarboxylic acid $(0.023 \mathrm{mmol})$ and $\mathrm{Pd}\left(\mathrm{PPh}_{3}\right)_{4}(0.0045 \mathrm{mmol})$ were added. The whole was heated under reflux conditions in an oil bath (oil bath temperature: $115^{\circ} \mathrm{C}$ ) for $10 \mathrm{~min}$. After the mixture was cooled to rt, water $(5 \mathrm{~mL})$ and brine $(5 \mathrm{~mL})$ were added. The organic materials were extracted with ethyl acetate, dried over sodium sulfate, passed through a short pad of silica gel, and concentrated under vacuum. The mixture was purified by silica gel preparative TLC $($ AcOEt/hexane $=1 / 10)$ to give $\mathbf{3 c}$ in $92 \%$ isolated yield $(56 \mathrm{mg})$. 


\section{tert-Butyl 2-oxo-1-(3-phenyl-2-propen-1-yl)-1-cyclopentanecarboxylate (3d)}

Cinnamyl alcohol (2a, $0.42 \mathrm{mmol})$ and tert-butyl 2-oxocyclopentane-1-carboxylate (1d, 0.28 mmol) were suspended in water $(1.7 \mathrm{~mL})$ at $\mathrm{rt}$ under argon, and then 1-adamantanecarboxylic acid $(0.028 \mathrm{mmol})$ and $\mathrm{Pd}\left(\mathrm{PPh}_{3}\right)_{4}(0.0056 \mathrm{mmol})$ were added. The whole was heated under reflux conditions in an oil bath (oil bath temperature: $115^{\circ} \mathrm{C}$ ) for $10 \mathrm{~min}$. After the mixture was cooled to $\mathrm{rt}$, water $(5 \mathrm{~mL})$ and brine $(5 \mathrm{~mL})$ were added. The organic materials were extracted with ethyl acetate, dried over sodium sulfate, passed through a short pad of silica gel, and concentrated under vacuum. The mixture was purified by silica gel preparative TLC $($ AcOEt/hexane $=1 / 5$, and then ether/hexane $=1 / 3)$ to give $\mathbf{3 d}$ in $76 \%$ isolated yield $(64 \mathrm{mg})$. Mp 69.4-69.5 ${ }^{\circ} \mathrm{C} ;{ }^{1} \mathrm{H}$ NMR $\left(300 \mathrm{MHz}, \mathrm{CDCl}_{3}\right) \delta 1.44(9 \mathrm{H}, \mathrm{s}), 1.84-2.06(3 \mathrm{H}, \mathrm{m}), 2.14-2.26$ $(1 \mathrm{H}, \mathrm{m}), 2.38-2.55(3 \mathrm{H}, \mathrm{m}), 2.75(1 \mathrm{H}, \mathrm{dd}, J=14.1,7.5 \mathrm{~Hz}), 6.10(1 \mathrm{H}, \mathrm{dt}, J=15.8,7.5 \mathrm{~Hz})$, $6.44(1 \mathrm{H}, \mathrm{d}, J=15.8 \mathrm{~Hz}), 7.18-7.34(5 \mathrm{H}, \mathrm{m}) ;{ }^{13} \mathrm{C} \mathrm{NMR}\left(75 \mathrm{MHz}, \mathrm{CDCl}_{3}\right) \delta 19.56,27.87$, $32.41,36.87,38.04,60.74,81.84,124.87,126.10,127.28,128.46,133.81,137.09,170.18$, 214.98; IR (film) 2975, 1749, 1721, 1368, 1251, $1144 \mathrm{~cm}^{-1}$; Anal. calcd. for $\mathrm{C}_{19} \mathrm{H}_{24} \mathrm{O}_{3}$ : C, 75.97; H, 8.05, found: C, 76.05; H, 8.13.

\section{Ethyl 2-methyl-2-nitro-5-phenylpent-4-enoate (3e)}

Cinnamyl alcohol (2a, $0.30 \mathrm{mmol})$ and ethyl 2-nitropropionate (1e, $0.20 \mathrm{mmol})$ were suspended in water $(1.2 \mathrm{~mL})$ at $\mathrm{rt}$ under argon, and then 1-adamantanecarboxylic acid (0.019 mmol) and $\mathrm{Pd}\left(\mathrm{PPh}_{3}\right)_{4}(0.0099 \mathrm{mmol})$ were added. The whole was heated under reflux conditions in an oil bath (oil bath temperature: $115^{\circ} \mathrm{C}$ ) for $1 \mathrm{~h}$. After the mixture was cooled to rt, water $(5 \mathrm{~mL})$ and brine $(5 \mathrm{~mL})$ were added. The organic materials were extracted with ethyl acetate, dried over sodium sulfate, passed through a short pad of silica gel, and concentrated under vacuum. The mixture was purified by silica gel preparative TLC $(\mathrm{AcOEt} / \mathrm{hexane}=1 / 10)$ to give $3 \mathbf{e}$ in $78 \%$ isolated yield $(40 \mathrm{mg}) .{ }^{1} \mathrm{H}$ NMR $(400 \mathrm{MHz}$, $\left.\mathrm{CDCl}_{3}\right) \delta 1.29(3 \mathrm{H}, \mathrm{t}, J=7.1 \mathrm{~Hz}), 1.81(3 \mathrm{H}, \mathrm{s}), 3.02(1 \mathrm{H}, \mathrm{dd}, J=14.4,7.6 \mathrm{~Hz}), 3.15(1 \mathrm{H}, \mathrm{dd}$, 
$J=14.4,7.3 \mathrm{~Hz}), 4.28(2 \mathrm{H}, \mathrm{q}, J=7.1 \mathrm{~Hz}), 6.02(1 \mathrm{H}, \mathrm{ddd}, J=15.6,7.6,7.3 \mathrm{~Hz}), 6.52(1 \mathrm{H}, \mathrm{d}$, $J=15.6 \mathrm{~Hz}), 7.23-7.34(5 \mathrm{H}, \mathrm{m}) ;{ }^{13} \mathrm{C} \mathrm{NMR}\left(100 \mathrm{MHz}, \mathrm{CDCl}_{3}\right) \delta 13.85,21.26,40.23,62.86$, $92.29,120.68,126.37,127.93,128.58,136.19,136.36,167.07$; IR (neat) 2984, 1749, 1553, 1255, $1130 \mathrm{~cm}^{-1}$; HRMS (EI), calcd. for $\mathrm{C}_{14} \mathrm{H}_{17} \mathrm{NO}_{4}: 263.1158$, found: 263.1129 .

\section{2-Benzenesulfonyl-1,5-diphenylpent-4-en-1-one (3f)}

Cinnamyl alcohol (2a, $0.26 \mathrm{mmol})$ and 2-benzenesulfonylacetophenone (1f, $0.23 \mathrm{mmol})$ were suspended in water $(1.4 \mathrm{~mL})$ at $\mathrm{rt}$ under argon, and then 1-adamantanecarboxylic acid $(0.024$ mmol $)$ and $\mathrm{Pd}\left(\mathrm{PPh}_{3}\right)_{4}(0.0047 \mathrm{mmol})$ were added. The whole was heated at $80{ }^{\circ} \mathrm{C}$ for $1.5 \mathrm{~h}$. After the mixture was cooled to $\mathrm{rt}$, water $(5 \mathrm{~mL})$ and brine $(5 \mathrm{~mL})$ were added. The organic materials were extracted with ethyl acetate, dried over sodium sulfate, passed through a short pad of silica gel, and concentrated under vacuum. The mixture was purified by silica gel column chromatography $($ AcOEt/hexane $=1 / 15-1 / 5)$ to give $\mathbf{3 f}$ in $>99 \%$ isolated yield $(88$ mg). Mp 115.3-115.4 ${ }^{\circ} \mathrm{C} ;{ }^{1} \mathrm{H}$ NMR (400 MHz, $\left.\mathrm{CDCl}_{3}\right) \delta 2.93-3.05(2 \mathrm{H}, \mathrm{m}), 5.21(1 \mathrm{H}, \mathrm{dd}, J=$ 10.2, 4.2 Hz), $5.92(1 \mathrm{H}, \mathrm{dt}, J=15.6,7.1 \mathrm{~Hz}), 6.40(1 \mathrm{H}, \mathrm{d}, J=15.6 \mathrm{~Hz}), 7.15-7.20(5 \mathrm{H}, \mathrm{m})$, 7.40-7.65 (6H, m), $7.80(2 \mathrm{H}, \mathrm{d}, J=7.6 \mathrm{~Hz}), 7.92(2 \mathrm{H}, \mathrm{d}, J=7.8 \mathrm{~Hz}) ;{ }^{13} \mathrm{C}$ NMR $(100 \mathrm{MHz}$, $\left.\mathrm{CDCl}_{3}\right) \delta 31.64,69.43,122.94,126.12,127.58,128.40,128.71,128.90,128.94,129.71$, 134.00, 134.14, 134.29, 136.29, 136.39, 136.93, 191.85; IR (film) 1679, 1448, 1309, 1148, 744, $688 \mathrm{~cm}^{-1}$; HRMS (EI), calcd. for $\mathrm{C}_{23} \mathrm{H}_{20} \mathrm{O}_{3} \mathrm{~S}: 376.1133$, found: 376.1153; Anal. calcd. for $\mathrm{C}_{23} \mathrm{H}_{20} \mathrm{O}_{3} \mathrm{~S}: \mathrm{C}, 73.38 ; \mathrm{H}, 5.35$, found: C, 73.11; H, 5.57.

\section{2,2-Dimethyl-4,6-dioxo-5,5-di(3-phenyl-2-propenyl)-1,3-dioxane (3g)}

Cinnamyl alcohol (2a, $0.49 \mathrm{mmol})$ and Meldrum's acid (1g, $0.23 \mathrm{mmol})$ were suspended in water $(1.3 \mathrm{~mL})$ at $\mathrm{rt}$ under argon, and then 1-adamantanecarboxylic acid $(0.022 \mathrm{mmol})$ and $\operatorname{Pd}\left(\mathrm{PPh}_{3}\right)_{4}(0.0011 \mathrm{mmol})$ were added. The whole was heated at $80{ }^{\circ} \mathrm{C}$ for $30 \mathrm{~min}$. After the mixture was cooled to rt, water $(5 \mathrm{~mL})$ and brine $(5 \mathrm{~mL})$ were added. The organic materials were extracted with ethyl acetate, dried over sodium sulfate, passed through a short pad of 
silica gel, and concentrated under vacuum. The mixture was purified by silica gel preparative TLC $($ AcOEt/hexane $=1 / 20)$ to give $\mathbf{3 g}$ in $74 \%$ isolated yield $(63 \mathrm{mg})$.

\section{2-(2-Propen-1-yl)-1,3-diphenylpropane-1,3-dione (3h)}

Allyl alcohol (2c, $0.42 \mathrm{mmol})$ and dibenzoylmethane (1a, $0.28 \mathrm{mmol})$ were suspended in water $(1.7 \mathrm{~mL})$ at $\mathrm{rt}$ under argon, and then 1-adamantanecarboxylic acid $(0.028 \mathrm{mmol})$ and $\operatorname{Pd}\left(\mathrm{PPh}_{3}\right)_{4}(0.014 \mathrm{mmol})$ were added. The whole was heated at $80{ }^{\circ} \mathrm{C}$ for $30 \mathrm{~min}$. After the mixture was cooled to rt, water $(5 \mathrm{~mL})$ and brine $(5 \mathrm{~mL})$ were added. The organic materials were extracted with ethyl acetate, dried over sodium sulfate, passed through a short pad of silica gel, and concentrated under vacuum. The mixture was purified by silica gel preparative TLC $($ ether/toluene $=1 / 25)$ to give $\mathbf{3 h}$ in $73 \%$ isolated yield $(54 \mathrm{mg})$.

\section{Benzyl 2-oxo-1-(2-propen-1-yl)-1-cyclopentanecarboxylate (3i)}

Allyl alcohol (2c, $0.40 \mathrm{mmol})$ and benzyl 2-oxocyclopentane-1-carboxylate (1h, $0.27 \mathrm{mmol})$ were suspended in water $(1.6 \mathrm{~mL})$ at $\mathrm{rt}$ under argon, and then 1-adamantanecarboxylic acid $(0.027 \mathrm{mmol})$ and $\mathrm{Pd}\left(\mathrm{PPh}_{3}\right)_{4}(0.013 \mathrm{mmol})$ were added. The whole was heated at $80{ }^{\circ} \mathrm{C}$ for 20 min. After the mixture was cooled to $\mathrm{rt}$, water $(5 \mathrm{~mL})$ and brine $(5 \mathrm{~mL})$ were added. The organic materials were extracted with ethyl acetate, dried over sodium sulfate, passed through a short pad of silica gel, and concentrated under vacuum. The mixture was purified by silica gel preparative TLC $(\mathrm{AcOEt} / \mathrm{hexane}=1 / 5)$ to give $\mathbf{3 c}$ in $93 \%$ isolated yield $(64 \mathrm{mg}) .{ }^{1} \mathrm{H}$ NMR $\left(300 \mathrm{MHz}, \mathrm{CDCl}_{3}\right) \delta$ 1.85-2.06 (3H, m), 2.17-2.29 (1H, m), 2.34-2.50 (3H, m), 2.69 $(1 \mathrm{H}, \mathrm{dd}, J=13.9,7.1 \mathrm{~Hz}), 5.04-5.11(2 \mathrm{H}, \mathrm{m}), 5.14(2 \mathrm{H}, \mathrm{s}), 5.60-5.74(1 \mathrm{H}, \mathrm{m}), 7.30-7.37(5 \mathrm{H}$, $\mathrm{m}) ;{ }^{13} \mathrm{C} \mathrm{NMR}\left(75 \mathrm{MHz}, \mathrm{CDCl}_{3}\right) \delta 19.44,32.04,37.77,38.02,59.92,66.98,119.13,127.85$, $128.19,128.50,132.83,135.52,170.70,214.23$; IR (neat) 2968, 1749, 1726, 1220, $1155 \mathrm{~cm}^{-1}$; HRMS (EI), calcd. for $\mathrm{C}_{16} \mathrm{H}_{18} \mathrm{O}_{3}: 258.1256$, found: 258.1302 .

\section{2-Hex-2-enyl-1,3-diphenylpropane-1,3-dione (3j)}


2-Hexen-1-ol (2d, $0.42 \mathrm{mmol})$ and dibenzoylmethane (1a, $0.28 \mathrm{mmol})$ were suspended in water $(1.7 \mathrm{~mL})$ at $\mathrm{rt}$ under argon, and then 1-adamantanecarboxylic acid $(0.028 \mathrm{mmol})$ and $\operatorname{Pd}\left(\mathrm{PPh}_{3}\right)_{4}(0.014 \mathrm{mmol})$ were added. The whole was heated at $80{ }^{\circ} \mathrm{C}$ for $2 \mathrm{~h}$. After the mixture was cooled to rt, water $(5 \mathrm{~mL})$ and brine $(5 \mathrm{~mL})$ were added. The organic materials were extracted with ethyl acetate, dried over sodium sulfate, passed through a short pad of silica gel, and concentrated under vacuum. The mixture was purified by silica gel preparative TLC $($ AcOEt/hexane $=1 / 15)$ to give $\mathbf{3 j}$ and $\mathbf{4}$ in $80 \%$ yield $(70 \mathrm{mg}, \mathbf{3 j}: \mathbf{4}=76: 4) .{ }^{1} \mathrm{H} \mathrm{NMR}$ $\left(300 \mathrm{MHz}, \mathrm{CDCl}_{3}\right) \delta 0.80(3 \mathrm{H}, \mathrm{t}, J=7.3 \mathrm{~Hz}), 1.27(2 \mathrm{H}$, sextet, $J=7.3 \mathrm{~Hz}), 1.86-1.92(2 \mathrm{H}, \mathrm{dt}$, $J=7.3,5.3 \mathrm{~Hz}), 2.82(2 \mathrm{H}, \mathrm{t}, J=6.2 \mathrm{~Hz}), 5.27(1 \mathrm{H}, \mathrm{t}, J=6.6 \mathrm{~Hz}), 5.45-5.50(2 \mathrm{H}, \mathrm{m}), 7.44$ $(4 \mathrm{H}, \mathrm{t}, J=7.5 \mathrm{~Hz}), 7.55(2 \mathrm{H}, \mathrm{t}, J=7.5 \mathrm{~Hz}), 7.96(4 \mathrm{H}, \mathrm{d}, J=7.5 \mathrm{~Hz}) ;{ }^{13} \mathrm{C} \mathrm{NMR}(75 \mathrm{MHz}$, $\left.\mathrm{CDCl}_{3}\right) \delta 13.52,22.29,32.63,34.42,57.33,126.40,128.55,128.78,133.38,133.44,136.10$ 195.73; IR (neat) 2958, 1695, 1671, 1597, 1449, $1268 \mathrm{~cm}^{-1}$; HRMS (EI), calcd. for $\mathrm{C}_{21} \mathrm{H}_{22} \mathrm{O}_{2}$ : 306.1620, found: 306.1626 .

\section{2-(1,3-diphenyl-2-propen-1-yl)-1,3-diphenylpropane-1,3-dione (3k)}

1,3-Diphenyl-2-propen-1-ol (2e, $0.26 \mathrm{mmol})$ and dibenzoylmethane (1a, $0.24 \mathrm{mmol})$ were suspended in water $(1.4 \mathrm{~mL})$ at $\mathrm{rt}$ under argon, and then 1-adamantanecarboxylic acid $(0.024$ mmol $)$ and $\mathrm{Pd}\left(\mathrm{PPh}_{3}\right)_{4}(0.012 \mathrm{mmol})$ were added. The whole was heated under reflux conditions in an oil bath (oil bath temperature: $115^{\circ} \mathrm{C}$ ) for $30 \mathrm{~min}$. After the mixture was cooled to rt, water $(5 \mathrm{~mL})$ and brine $(5 \mathrm{~mL})$ were added. The organic materials were extracted with ethyl acetate, dried over sodium sulfate, passed through a short pad of silica gel, and concentrated under vacuum. The mixture was purified by silica gel column chromatography $(\mathrm{AcOEt} / \mathrm{hexane}=1 / 20)$ to give $3 \mathbf{k}$ in $88 \%$ isolated yield $(88 \mathrm{mg}) . \mathrm{Mp} 132.4-133.4{ }^{\circ} \mathrm{C} ;{ }^{1} \mathrm{H}$ NMR $\left(400 \mathrm{MHz}, \mathrm{CDCl}_{3}\right) \delta 4.80(1 \mathrm{H}, \mathrm{dd}, J=10.5,6.8 \mathrm{~Hz}), 5.95(1 \mathrm{H}, \mathrm{d}, J=10.5 \mathrm{~Hz}), 6.26-$ $6.36(2 \mathrm{H}, \mathrm{m}), 7.07-7.24(8 \mathrm{H}, \mathrm{m}), 7.31-7.35(4 \mathrm{H}, \mathrm{m}), 7.42-7.48(3 \mathrm{H}, \mathrm{m}), 7.55(1 \mathrm{H}, \mathrm{t}, J=7.3$ $\mathrm{Hz}), 7.82(2 \mathrm{H}, \mathrm{d}, J=7.3 \mathrm{~Hz}), 8.03(2 \mathrm{H}, \mathrm{d}, J=7.3 \mathrm{~Hz}) ;{ }^{13} \mathrm{C} \mathrm{NMR}\left(75 \mathrm{MHz}, \mathrm{CDCl}_{3}\right) \delta 49.98$, 
$62.56,126.20,126.83,127.29,128.26,128.47,128.55,128.60,128.74,128.85,129.86$, 131.80, 133.19, 133.41, 136.81, 136.84, 137.21, 140.88, 193.70, 194.36; IR (KBr) 1688, 1595, 1494, 1447, 1260, 1201, 965, 744, $686 \mathrm{~cm}^{-1}$; HRMS (EI), calcd. for $\mathrm{C}_{30} \mathrm{H}_{24} \mathrm{O}_{2}: 416.1776$, found: 416.1736; Anal. calcd. for $\mathrm{C}_{30} \mathrm{H}_{24} \mathrm{O}_{2}$ : C, 86.51; H, 5.81, found: C, 86.30; H, 6.02.

Plot of yield vs. time for the reactions of $1 b$ with $2 \mathrm{a}$ or $2 \mathrm{~b}$ at $100{ }^{\circ} \mathrm{C}$ in the presence of 1 $\mathrm{AdCO}_{2} \mathrm{H}$ in water

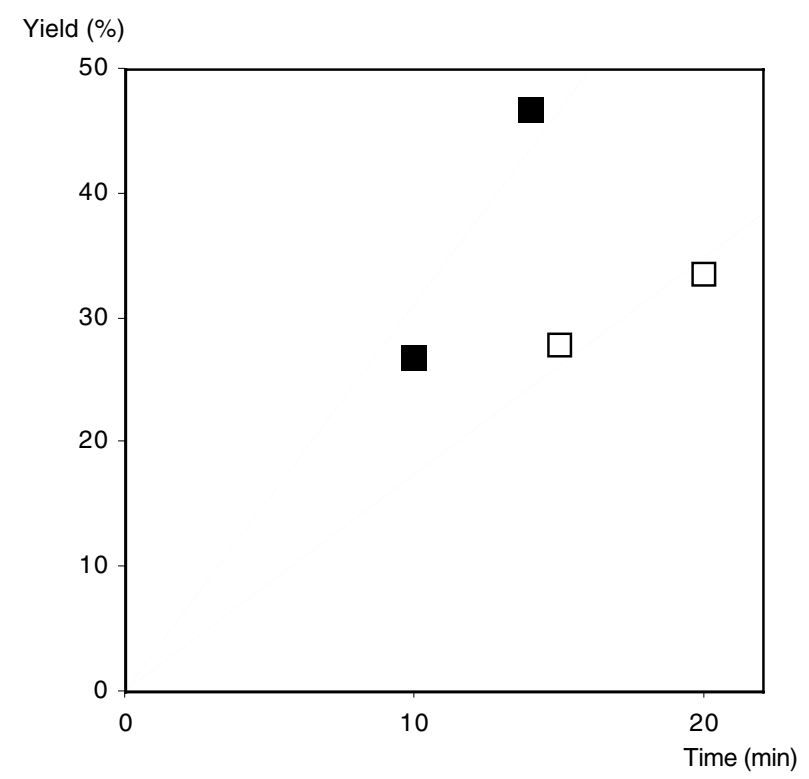

Conditions: $\mathrm{Pd}\left(\mathrm{PPh}_{3}\right)_{4}(2 \mathrm{~mol} \%), 1-\mathrm{AdCO}_{2} \mathrm{H}(10 \mathrm{~mol} \%), \mathbf{1 b}: \mathbf{2 a}(\mathbf{2 b})=1: 1.5$, at $100{ }^{\circ} \mathrm{C}$ in water. Open square: $\mathbf{1 b}+\mathbf{2 a}$, closed square: $\mathbf{1 b}+\mathbf{2 b}$.

\section{References}

[1] M. Sekiya, K. Suzuki, Chem. Pharm. Bull. 1970, 18, 1530.

[2] Y. Masuyama, K. Yamada, S. Shimizu, Y. Kurusu, Bull. Chem. Soc. Jpn. 1989, 62, 2913.

[3] M. Prat, M. Moreno-Manas, J. Ribas, Tetrahedron 1988, 44, 7205.

[4] J. R. Hwu, C. N. Chen, S.-S. Shiao, J. Org. Chem. 1995, 60, 856. 